\title{
La terapia del diabete nel paziente nefropatico
}

\author{
Edoardo Mannucci, Ilaria Dicembrini \\ Diabetologia, Azienda Ospedaliero-Universitaria Careggi, Firenze
}

\begin{abstract}
Diabetes therapies in renal impairment
Diabetes mellitus is recognized as a leading cause of chronic kidney disease and end-stage renal failure. Until few years ago patients with diabetes should be preferably treated with insulin. The management of the patients with hyperglycemia and chronic renal failure demands a close collaboration between the diabetologist and the nephrologists. Some of the new drugs as the Dipeptydil Peptidase-4 inhibitors and GLP-1 receptor agonists recently approved for the treatment of diabetes can be used, even if with a reduction of the usual dose, in chronic kidney disease and end-stage renal failure. These drugs showed efficacy on the improvement of HbA1c, several extraglycemic effects together with a low hypoglycemic risk.

This article will analyze current status of national and international guidelines and the product characteristics focusing on the treatment of diabetes in patients with impairment of renal function.
\end{abstract}

Keywords: GLP-1 receptor agonists, SGLT-2 inhibitors, Renal impairment

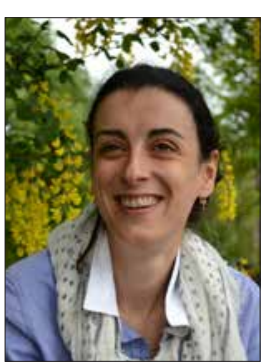

Ilaria Dicembrini

\section{Introduzione}

La terapia del diabete mellito tipo 2 è stata, per molto tempo, affidata a un numero limitato di farmaci: I'insulina (introdotta negli anni '30) e, a partire dagli anni '50, sulfaniluree e metformina. A parte l'acarbose, che, peraltro, ha avuto una diffusione limitata, è stato necessario attendere fino agli anni '90 per avere una reale novità, rappresentata dai tiazo-

lidinedioni. Poi, nell'ultimo decennio, c'è stata una vera e propria esplosione di novità farmacologiche, con l'introduzione di ben dodici farmaci appartenenti a tre classi diverse: agonisti GLP-1, inibitori DPP4 e inibitori SGLT-2.

Gli agonisti recettoriali del GLP-1 sfruttano le proprietà terapeutiche di un ormone peptidico (il Glucagon-Like Peptide-1 o GLP-1), prodotto a livello intestinale prevalentemente nella fase post-prandiale, che stimola la secrezione di insulina e inibisce quella di glucagone in maniera glucosio-dipenden-

Accepted: April 30, 2015

Published online: June 17, 2015

Indirizzo per la corrispondenza:

Dr. Edoardo Mannucci

Diabetologia, Padiglione 28C

Azienda Ospedaliero-Universitaria Careggi

Via delle Oblate 4

50134 Firenze

edoardo.mannucci@unifi.it te; ciò significa che il GLP-1 è in grado di ridurre la glicemia se è elevata, mentre è privo di effetti se il glucosio è normale, restando virtualmente privo di rischio ipoglicemico, a meno che non venga combinato con una sulfanilurea o con l'insulina. Inoltre, a concentrazioni soprafisiologiche, il GLP1-1 è in grado di inibire l'assunzione di cibo, inducendo un calo ponderale. Purtroppo, il GLP-1 umano non può essere utilizzato come farmaco, in quanto ha una cinetica sfavorevole: viene rapidamente inattivato da varie peptidasi, tra le quali la più importante è la dipeptidil-peptidasi-4 (DPP4), tanto che la sua emivita dopo somministrazione endovenosa o sottocutanea è di pochissimi minuti. Per questo motivo, sono stati sviluppati agonisti specifici del recettore del GLP-1 resistenti alla DPP4, la cui emivita è compatibile con l'uso terapeutico. Le molecole di questa classe sono tutte somministrate per via sottocutanea, ma differiscono ampiamente tra loro per cinetica, durata d'azione e frequenza di somministrazione (due volte al giorno per exenatide, una volta al giorno per lixisenatide e liraglutide e una volta alla settimana per exenatide LAR). Le molecole a più lunga durata d'azione (liraglutide ed exenatide LAR) hanno maggiore efficacia sulla glicemia a digiuno e sull'emoglobina glicata, mentre quelle più rapide (exenatide $\mathrm{e}$ lixisenatide) sono più efficaci sull'iperglicemia post-prandiale (1). Tutte le molecole inducono anche un rilevante calo ponderale (2), per cui alcune di esse saranno presto disponibili anche come terapia dell'obesità non associata al diabete.

Una strategia alternativa per sfruttare il potenziale terapeutico del GLP-1 è quello di prolungarne l'emivita attraverso l'inibizione della DPP4. Esistono in commercio, attualmente, 5 diversi inibitori della DPP4, tutti somministrabili oralmente una o due volte al giorno. Questi farmaci, che incrementa- 
no le concentrazioni circolanti del GLP-1 endogeno, agiscono soprattutto sull'iperglicemia post-prandiale e sono, nel complesso, meno efficaci rispetto agli agonisti del GLP-1. Inoltre, essendo il livello di stimolazione dell'asse del GLP-1 che si può ottenere con gli inibitori DPP4 meno marcato rispetto agli agonisti recettoriali, con questi farmaci non si osserva una riduzione di peso.

Una novità maggiore degli ultimissimi anni è rappresentata dagli inibitori SGLT-2, che riducono il riassorbimento del glucosio nel tubulo prossimale, abbassando la soglia renale e inducendo glicosuria. L'entità della perdita giornaliera di glucosio è indipendente dalla glicemia e correlata alla filtrazione glomerulare. La glicosuria ha l'effetto di ridurre l'iperglicemia; peraltro, questi farmaci non sono in grado di indurre ipoglicemia, a meno che non siano associati a insulina o sulfaniluree, in quanto la perdita di glucosio urinario in condizioni di normoglicemia si associa a un aumento della gluconeogenesi epatica. Il principale effetto collaterale degli inibitori SGLT2, che possono essere usati sia in monoterapia che in associazione con altri farmaci orali o con l'insulina, è rappresentato dalle infezioni genitali e, in particolare, dalla Candida.

L'introduzione delle nuove classi di farmaci ha modificato gli algoritmi abituali per la terapia del diabete tipo 2. Ciò vale anche per i pazienti diabetici con insufficienza renale, nei quali la gamma di opzioni terapeutiche disponibili si è notevolmente ampliata grazie all'introduzione dei nuovi farmaci.

\section{Nefropatia, cinetica, efficacia e tossicità dei farmaci per il diabete}

\section{Metformina}

La metformina, farmaco di prima scelta nella terapia del diabete tipo 2, viene assunta per via orale, assorbita a livello intestinale ed escreta immodificata mediante ultrafiltrazione glomerulare e secrezione tubulare. Circa il 90\% della dose è eliminato nelle successive $12-24$ ore. La sua emivita è compresa tra 1.5 e 9 ore. Pur non essendo un farmaco nefrotossico, in caso di compromissione della funzione renale si può verificare un accumulo di metformina. L'evento più temibile è rappresentato dallo sviluppo di acidosi lattica, che, pur essendo un'eventualità rara (5/100.000 pazienti/ anno), è gravato da una mortalità superiore al $40 \%$. In base alla scheda tecnica italiana, il farmaco risulta controindicato per un tasso stimato di filtrazione glomerulare (eGFR) $<60 \mathrm{~mL} / \mathrm{min} / 1.73 \mathrm{~m}^{2}$ (3). Anche le Linee Guida della National Kidney Foundation pongono delle precise limitazioni all'uso della metformina in caso di compromissione della funzione renale, controindicandola in caso di livelli sierici di creatinina rispettivamente pari o superiori a $1.4 \mathrm{mg} / \mathrm{dL}$ nelle femmine e a 1.5 nei maschi. Tuttavia, una revisione sistematica di tutti gli studi clinici di confronto e osservazionali di coorte ha mostrato che il rischio di acidosi lattica associato a metformina potrebbe essere sopravvalutato (4). Le Linee
Guida del National Institute for Health and Clinical Excellence (NICE) (5) consigliano di rivedere il dosaggio di metformina se l'eGFR è $<45 \mathrm{~mL} / \mathrm{min} / 1.73 \mathrm{~m}^{2}$ e di interrompere il trattamento solo per valori $<30$. In tutte le condizioni con eGFR $<60 \mathrm{~mL} / \mathrm{min} / 1.73 \mathrm{~m}^{2}$, la stima del filtrato glomerulare renale deve essere eseguita non più con cadenza annuale, ma semestralmente. Al tempo stesso, anche la necessità di sospendere il farmaco per 48 ore in caso di indagini con mezzo di contrasto per via endovenosa appare ridimensionata e riservata esclusivamente ai pazienti con eGFR $<60$ $\mathrm{mL} / \mathrm{min} / 1.73 \mathrm{~m}^{2}$.

\section{Sulfaniluree e glinidi}

L'uso delle sulfaniluree, considerati il costo, la somministrazione per via orale e l'efficacia almeno nel breve termine, appare ancora diffuso. Purtroppo, anche l'utilizzo delle molecole di seconda generazione (glibenclamide, glimepiride, gliclazide e glipizide), con un'emivita minore rispetto alle molecole di prima generazione, appare sempre gravato dal rischio di ipoglicemia, rischio che risulta ulteriormente aumentato in presenza di insufficienza renale (6). I pazienti con compromissione moderata-grave della funzione renale (eGFR $<60 \mathrm{~mL} / \mathrm{min} / 1.73 \mathrm{~m}^{2}$ ) hanno infatti, di per sé, un aumentato rischio di ipoglicemia legato principalmente a due condizioni:

1) la compromissione della clearance dell'insulina e di alcuni antidiabetici orali

2) la riduzione della gluconeogenesi renale

Tra le sulfaniluree di seconda generazione, soltanto glipizide e gliclazide non hanno metaboliti attivi eliminati per via renale, al contrario della glimepiride che, pur essendo metabolizzata a livello epatico, ha metaboliti attivi eliminati dal rene. L'utilizzo di tutte le sulfaniluree (7), in accordo con quanto suggerito dalla National Kidney Foundation, dovrebbe essere evitato in caso di eGFR $<30 \mathrm{~mL} / \mathrm{min} / 1.73 \mathrm{~m}^{2}$ (8). Anche repaglinide, un farmaco appartenente sempre alla classe dei secretagoghi prevalentemente metabolizzato a livello epatico, con un'emivita di 0.6 -1.8 ore ed escrezione mediante bile e feci, considerando che viene eliminato soltanto per circa l'8\% della dose per via renale, non richiede alcun aggiustamento posologico almeno in caso di compromissione del filtrato glomerulare lieve-moderata (9).

\section{Inibitori $\alpha$-glucosidasi}

L'acarbose, come la metformina, non ha effetti negativi sul peso corporeo e non aumenta il rischio di ipoglicemia. Il profilo di tollerabilità di questo farmaco è limitato dall'insorgenza di possibili effetti collaterali gastrointestinali. In accordo con la scheda tecnica del farmaco, il suo utilizzo è indicato per valori di eGFR >25 mL/min/1.73 $\mathrm{m}^{2}(10)$. 


\section{Pioglitazone}

Il pioglitazone, somministrato per via orale, viene completamente metabolizzato a livello epatico, con la formazione di sei metaboliti, di cui tre attivi. L'emivita plasmatica del farmaco immodificato è di 5-6 ore e quella dei suoi metaboliti è di 16-23 ore. In caso di insufficienza renale, non si verifica accumulo né del farmaco né dei suoi prodotti di metabolizzazione (11). Anzi, nei pazienti con insufficienza renale, le concentrazioni plasmatiche di pioglitazone e dei suoi metaboliti sono inferiori rispetto a quelle osservate in soggetti con funzionalità renale normale, probabilmente per la riduzione del legame con le proteine plasmatiche. La scheda tecnica italiana del farmaco ne autorizza il trattamento senza alcun aggiustamento posologico per clearance della creatinina $>4 \mathrm{~mL} / \mathrm{min}$ (11). Va tuttavia ricordato che i pazienti con compromissione della funzione renale spesso presentano un quadro di ritenzione idrica, che può essere aggravata dal trattamento con tiazolidinedioni (9).

\section{Insulina}

Teoricamente, l'insulina può essere somministrata in qualsiasi stadio di insufficienza renale. Il suo profilo di efficacia risulta tuttavia gravato da un aumento del rischio di episodi ipoglicemici, rischio, come abbiamo già visto, ulteriormente aumentato in caso di insufficienza renale, e, pertanto, dalla necessità di un regolare monitoraggio glicemico domiciliare e di un'adeguata educazione del paziente. Nonostante sia documentata la presenza di una compromissione della sensibilità periferica tissutale all'insulina, in caso di insufficienza renale si verifica una riduzione del fabbisogno insulinico giornaliero. In primo luogo, l'insulina somministrata per via esogena ha una metabolizzazione prevalentemente renale, a differenza di quella endogena metabolizzata prevalentemente a livello epatico (9). Tutta l'insulina somministrata viene filtrata a livello glomerulare e poi riassorbita a livello del tubulo prossimale. Esiste anche un meccanismo di riassorbimento ad opera delle cellule peritubulari che va incontro a un aumento compensatorio al ridursi del filtrato glomerulare, almeno fino a valori superiori a $20 \mathrm{~mL} /$ $\mathrm{min} / 1.73 \mathrm{~m}^{2}$ (12). Per forme severe di compromissione della funzione renale si verifica una riduzione della metabolizzazione con conseguente aumento dell'emivita plasmatica sia degli analoghi a breve durata sia di quelli a lunga durata d'azione. Viene pertanto suggerita una riduzione del $25 \%$ della dose insulinica abituale in caso di eGFR $<50$ e $>30 \mathrm{~mL} /$ $\mathrm{min} / 1.73 \mathrm{~m}^{2}$ e del $50 \%$ per valori $<10 \mathrm{~mL} / \mathrm{min} / 1.73 \mathrm{~m}^{2}$ (13). Tale riduzione della dose insulinica abituale risente anche del trattamento emodialitico e di dialisi peritoneale. In entrambe queste condizioni si verifica un miglioramento della sensibilità insulinica con un controllo glicemico migliore a dosi inferiori di insulina. La somministrazione in particolare di insulina nella cavità peritoneale vuota sembra essere as- sociata a una riduzione del fabbisogno insulinico, mentre la sua somministrazione insieme al liquido di dialisi ne riduce l'attività.

Alla luce di queste considerazioni, qualora in un paziente con insufficienza renale sia indicato il trattamento insulinico, è da suggerire uno schema intensivo basal-bolus che preveda l'utilizzo di analoghi a breve e a lunga durata d'azione. Le schede tecniche italiane degli analoghi a lunga durata d'azione attualmente disponibili (glargine, detemir e la più recente degludec) sottolineano l'assenza di differenze nella farmacocinetica in presenza di insufficienza renale, ma sottolineano la necessità di un adeguamento posologico in relazione alle necessità individuali (14-18). Tali indicazioni vengono riportate anche per gli analoghi a breve durata d'azione (lispro, aspart e glulisine) (19-21).

\section{Inibitori della Dipeptidil peptidasi-4 (DPP-4)}

Attualmente, sono disponibili 5 molecole (sitagliptin, vildagliptin, saxagliptin, alogliptin e linagliptin), la cui prescrizione come monoterapia è rimborsabile soltanto in caso di insufficienza renale, previo adeguamento posologico (fatta eccezione per il linagliptin) per le forme non lievi. Le prime 4 molecole hanno, infatti, un'eliminazione prevalentemente renale, mentre linagliptin viene escreto quasi interamente per via biliare. Questo determina un'esposizione al farmaco direttamente correlata al declino dell'eGFR. L'adeguamento posologico in relazione al grado di compromissione del GFR prevede un dimezzamento progressivo della dose per valori compresi tra 50 e $30 \mathrm{~mL} / \mathrm{min} / 1.73 \mathrm{~m}^{2}$ e inferiori a $30 \mathrm{~mL} /$ $\mathrm{min} / 1.73 \mathrm{~m}^{2}$ (22). Tutte le molecole hanno prove di efficacia e sicurezza su pazienti con insufficienza renale moderata, grave e terminale \pm dialisi. Sitagliptin ha prove di efficacia e sicurezza anche nei trapiantati di rene che successivamente sviluppano diabete tipo 2 (23).

\section{Agonisti recettoriali del GLP-1}

Attualmente, sono disponibili 4 molecole di questa classe: exenatide, liraglutide, exenatide LAR e lixisenatide. Presentano marcate differenze di farmacocinetica, tanto da poter essere distinti in agonisti recettoriali a breve durata d'azione (exenatide e lixisenatide) e a lunga durata d'azione (exenatide LAR e liragutide). Questi farmaci devono essere iniettati a livello sottocutaneo con una frequenza variabile da quella bigiornaliera (exenatide) a 1 volta a settimana (exenatide LAR). Attualmente, le schede tecniche delle molecole per exenatide ne consigliano la riduzione della posologia per forme moderate di insufficienza renale, sia per exenatide che per lixisenatide non ne raccomandano l'uso nei pazienti con forme gravi e terminali e per exenatide LAR ne sconsigliano l'uso nei pazienti con compromissione della funzione renale. Per liraglutide ne viene sconsigliato l'utilizzo soltanto nelle forme gravi e terminali. D'altra parte, exenatide viene 
eliminata mediante filtrazione glomerulare e degradazione proteolitica a livello renale, mentre liraglutide ha una farmacocinetica indipendente dalla funzione renale (24). Lixisenatide ed exenatide LAR (22) hanno, invece, dati limitati o assenti su pazienti con insufficienza renale moderata e grave.

\section{Inibitori del riassorbimento tubulare del glucosio (SGLT-2 inibitori)}

Il rene rappresenta il bersaglio di azione dell'ultima classe di antidiabetici attualmente disponibile. Gli inibitori del cotrasportatore sodio-glucosio 2 (SGLT-2) agiscono su queste specifiche proteine di membrana, localizzate prevalentemente a livello del tubulo contorto prossimale, dove sono responsabili del riassorbimento di circa il 90-95\% del glucosio. Le molecole attualmente disponibili (canaglifozin, dapaglifozin, empaglifozin) hanno un profilo farmacocinetico simile con rapido assorbimento e un'emivita plasmatica di 16-18 ore. Per effetto della glicosuria, questi farmaci presentano anche interessanti effetti extrarenali, come il calo ponderale, e, per effetto della diuresi osmotica, una riduzione persistente della pressione arteriosa sistolica e diastolica. Malgrado ciò, la loro azione antidiabetica risulta direttamente correlata al filtrato glomerulare renale con un'efficacia compromessa in presenza di insufficienza renale moderata, grave e terminale (25).

Il loro utilizzo non viene raccomandato in presenza di eGFR < a $60 \mathrm{~mL} / \mathrm{min} / 1.73 \mathrm{~m}^{2}$; ciò dipende dalla ridotta efficacia e non da problemi di sicurezza. Peraltro, nei pazienti in cui inizia un trattamento con SGLT-2 inibitori, si osserva un lieve decremento dell'eGFR (nell'ordine di 4-5 mL/min), reversibile con la sospensione del farmaco e probabilmente attribuibile alla deplezione di volume e alla riduzione del flusso ematico renale (Tab. I).

\section{Esistono farmaci con aspetti nefroprotettivi specifici?}

Il pioglitazone rappresenta una classe di antidiabetici orali che esercita un'azione ipoglicemizzante mediante attivazione di uno specifico tipo di recettori nucleari con conseguente miglioramento dell'insulino-resistenza. Questa classe, al di là del miglioramento del compenso glicemico, ha mostrato anche un'ampia gamma di effetti extraglicemici. Oltre a benefici su diverse componenti della sindrome metabolica e su alcuni marker di rischio cardiovascolare, alcune evidenze sembrano suggerire un possibile effetto protettivo sul versante renale. Una metanalisi, che ha incluso studi clinici randomizzati della durata di almeno 3 mesi, ha riscontrato come, sia nei pazienti normo- o micro-albuminurici sia nei macro-albuminurici o con franca proteinuria, il trattamento con tiazolidinedioni si associ a una significativa riduzione della proteinuria (26). Questi risultati sembrano supportati da numerose evidenze sperimentali che ipotizzano un possibile meccanismo d'azione sia diretto (visto che i recettori PPAR-Y sono presenti a livello glomerulare e tubulare) che indiretto (mediante miglioramento del controllo glicemico e pressorio, dello stato infiammatorio,

TABELLA I - Tabella riepilogativa degli attuali farmaci antidiabetici per il trattamento del diabete in relazione al grado di funzione renale (Creatinina Clearance; $\mathrm{mL} / \mathrm{min}$ )

\begin{tabular}{|c|c|c|c|c|}
\hline & $>60$ & $30-50$ & $<30$ & Dialisi \\
\hline \multicolumn{5}{|l|}{ Metformina } \\
\hline \multicolumn{5}{|l|}{ Sulfaniluree } \\
\hline \multicolumn{5}{|l|}{ Glinidi } \\
\hline \multicolumn{5}{|l|}{ Acarbose } \\
\hline \multicolumn{5}{|l|}{ Pioglitazone } \\
\hline \multicolumn{5}{|c|}{ Sitagliptin, Saxagliptin, Vildagliptin, Alogliptin } \\
\hline \multicolumn{5}{|l|}{ Linagliptin } \\
\hline \multicolumn{5}{|l|}{ Exenatide } \\
\hline \multicolumn{5}{|l|}{ Liraglutide } \\
\hline \multicolumn{5}{|l|}{ Lixisenatide } \\
\hline \multicolumn{5}{|l|}{ Exenatide LAR } \\
\hline Insulina & & & & \\
\hline
\end{tabular}

Grigio = indicato senza adeguamento posologico; Rosso = indicato con adeguamento posologico o uso con precauzione; Rosso scuro = controindicato. 
dello stress ossidativo e della funzione endoteliale). Anche una sotto-analisi dello studio clinico DREAM (Diabetes Reduction Assessment with ramipril and rosiglitazone Medication) ha confermato come il trattamento con tiazolidinedioni si associ a una riduzione del $20 \%$ della progressione del danno renale (da normo- a microalbuminuria e da micro- a proteinuria, riduzione del valore di eGFR $\geq 30 \%$ o progressione a insufficienza renale terminale) (26).

Malgrado tutte queste evidenze indirette, dobbiamo sottolineare che a oggi non si ha una chiara dimostrazione di un possibile effetto nefroprotettivo da parte dei tiazolidinedioni. Infatti non risultano ancora realizzati degli studi clinici a lungo termine aventi come obiettivo primario quello di valutare concreti benefici renali (come l'incidenza di insufficienza renale terminale) nei pazienti diabetici con nefropatia trattati con pioglitazone.

Per gli inibitori DPP-4, sitagliptin (27), linagliptin $(28,29)$ e saxagliptin (30) hanno mostrato, mediante studi clinici randomizzati, una riduzione dell'escrezione urinaria di albumina nei pazienti affetti da diabete mellito tipo 2 con o senza compromissione della funzione renale. Studi clinici specificatamente designati per verificarne i potenziali effetti nefroprotettivi sono tuttora in corso $(31,32)$.

I meccanismi mediante i quali gli inibitori della DPP-4 si associano a una riduzione dell'escrezione urinaria di albumina potrebbero essere legati a effetti specifici a livello renale di miglioramento del grado di stress ossidativo, disfunzione endoteliale e stato flogistico (33).

\section{Conclusioni}

Nonostante alcune incoraggianti osservazioni per taluni farmaci, a oggi non possiamo affermare con certezza che molecole impiegate per la terapia del diabete abbiano specifici effetti nefroprotettivi, come accade invece per alcune classi di antipertensivi. Inoltre, l'insufficienza renale resta un'importante limitazione all'utilizzo di molti dei farmaci più diffusi per il diabete, a partire dalla metformina. Nonostante ciò, l'ampliamento delle classi farmacologiche consente di utilizzare una gamma crescente di molecole anche nei pazienti con insufficienza renale. Come conseguenza di ciò, molte persone che in passato avrebbero potuto essere gestite solo con l'insulina oggi possono raggiungere un buon compenso glico-metabolico anche soltanto con farmaci non insulinici. Se ciò, da un lato, complica l'impostazione terapeutica, dall'altro lato offre occasioni migliori di cura per numeri crescenti di pazienti.

\section{Disclosures}

Financial support: No financial support was received for this submission. Conflict of interest: E.M. received on the last 5 years consultancy fees from Merck Serono, Merck Sharp Dohme, Bristol Mayers-Squibb, Eli Lilly, Glaxo Simthkline, Jannsen, takeda, Novo Nordisk, Sanofi and Takeda. I.D. has no conflicts of interest to declare.

\section{Bibliografia}

1. Lindamood CA, Taylor JR. Emerging New Therapies for the Treatment of Type 2 Diabetes Mellitus: Glucagon-like Peptide-1 Receptor Agonists. Clin Ther. 20151;37(3):483-93.

2. Monami M, Dicembrini I, Marchionni N, Rotella CM, Mannucci E. Effects of glucagon-like peptide-1 receptor agonists on body weight: a meta-analysis. Exp Diabetes Res. 2012;2012:672658.

3. https://farmaci.agenziafarmaco.gov.it/aifa/servlet/PdfDownlo adServlet?pdfFileName=footer 000898039066 RCP.

4. Salpeter SR, Greyber E, Pasternak GA, Salpeter EE. Risk of fatal and nonfatal lactic acidosis with metformin use in type 2 diabetes mellitus. Cochrane Database Syst Rev. 2010;(4): CD002967.

5. National Institute for Health and Care Excellence. The management of type 2 diabetes (CG87). 2009. http://guidance. nice.org.uk/cg87 (accessed October 10, 2013).

6. Schernthaner G. Kidney disease in diabetology: lessons from 2008. Nephrol Dial Transplant. 2009;24(2):396-9.

7. http://www.ema.europa.eu/docs/en GB/document library/ EPAR_-_Product_Information/human/000680/WC500033442.pdf.

8. National Kidney Foundation. KDOQI Clinical Practice Guideline for Diabetes and CKD: 2012 Update. Am J Kidney. Dis 2012;60(5):850-86.

9. Avogaro A, Schernthaner G. Achieving glycemic control in patients with type 2 diabetes and renal impairment. Acta Diabetol. 2013;50(3):283-91.

10. https://farmaci.agenziafarmaco.gov.it/aifa/servlet/PdfDownlo adServlet?pdfFileName=footer_002392_038835_RCP.

11. https://farmaci.agenziafarmaco.gov.it/aifa/servlet/PdfDownlo adServlet?pdfFileName=footer_000712_034946_RCP.

12. Biesembach G, Raml A, Schmekal B, Eichbauer-Sturm G. Decreased insulin requirement in relation to GFR in nephropatic type 1 and insulin-treated type 2 diabetic patients. Diabet Med. 2003;20:642-5.

13. Trevisan $R$, Dodesini $A R$, Lepore G. Terapia del diabete nel paziente nefropatico. G It Diabetol Metab 2015; 35:27-35.

14. https://farmaci.agenziafarmaco.gov.it/aifa/servlet/PdfDown loadServlet?pdfFileName=footer_001368_035724_RCP.

15. https://farmaci.agenziafarmaco.gov.it/aifa/servlet/PdfDown loadServlet?pdfFileName=footer_000085_036850_RCP.

16. http://www.ema.europa.eu/docs/en_GB/document_library/ EPAR_-_Product_Information/human/000284/WC500036082.pdf.

17. http://www.ema.europa.eu/docs/en_GB/document_library/ EPAR_-_Product_Information/human/000528/WC500036662.pdf.

18. http://www.ema.europa.eu/docs/en_GB/document_library/ EPAR_-_Product_Information/human/002498/WC500138940.pdf.

19. https://farmaci.agenziafarmaco.gov.it/aifa/servlet/PdfDo wnloadServlet?pdfFileName=footer_001230_033637_RCP. pdf\&retry $=0 \&$ sys $=m 0 b 13$.

20. https://farmaci.agenziafarmaco.gov.it/aifa/servlet/PdfDownlo adServlet?pdfFileName=footer_001368_036684_RCP.

21. https://farmaci.agenziafarmaco.gov.it/aifa/servlet/PdfDownlo adServlet?pdfFileName=footer_000085_034498_RCP.

22. Scheen AJ. Pharmacokinetics and clinical use of incretin-based therapies in patients with chronic kidney disease and type 2 diabetes. Clin Pharmacokinet. 2015;54(1): 1-21.

23. Strøm Halden TA, Åsberg A, Vik K, Hartmann A, Jenssen T. Short-term efficacy and safety of sitagliptin treatment in longterm stable renal recipients with new-onset diabetes after transplantation. Nephrol Dial Transplant. 2014;29(4):926-33.

24. Jacobsen LV, Hindsberger C, Robson R, Zdravkovic M. Effect of renal impairment on the pharmacokinetics of the GLP1 analogue liraglutide. $\mathrm{Br} J$ Clin Pharmacol. 2009;68(6): 898-905. 
25. Kasichayanula S, Liu X, Pe Benito $M$, et al. The influence of kidney function on dapagliflozin exposure, metabolism and pharmacodynamics in healthy subjects and in patients with type 2 diabetes mellitus. Br J Clin Pharmacol. 2013;76(3): 432-44.

26. Sarafidis PA, Stafylas PC, Georgianos PI, Saratzis AN, Lasaridis AN. Effect of thiazolidinediones on albuminuria and proteinuria in diabetes: a meta-analysis. Am J Kidney Dis. 2010;55(5): 835-47.

27. Mori H, Okada Y, Arao T, Tanaka Y. Sitagliptin improves albuminuria in patients with type 2 diabetes mellitus. J Diabetes Investig. 2014;5(3):313-9.

28. Groop PH, Del Prato S, Taskinen MR, et al. Linagliptin treatment in subjects with type 2 diabetes with and without mild-tomoderate renal impairment. Diabetes Obes Metab. 2014;16 (6):560-8
29. Groop PH, Del Prato S, Taskinen MR, et al. Linagliptin treatment in subjects with type 2 diabetes with and without mild-to-moderate renal impairment. Diabetes Obes Metab. 2014;16(6):560-8.

30. Udell JA, Bhatt DL, Braunwald E, et al. Saxagliptin and Cardiovascular Outcomes in Patients With Type 2 Diabetes and Moderate or Severe Renal Impairment: Observations From the SAVOR-TIMI 53 Trial. Diabetes Care. 2015;38(4):696-705.

31. MARLINA (Efficacy, Safety \& Modification of Albuminuria in Type 2 Diabetes Subjects with Renal Disease with LINAgliptin) trial (NCT01792518).

32. CARMELINA (CArdiovascular Safety \& Renal Microvascular OutcomE Study with LINAgliptin) trial (NCT01897532).

33. Haluzík M, Frolík J, Rychlík I. Renal Effects of DPP-4 Inhibitors: A Focus on Microalbuminuria. Int J Endocrinol. 2013;2013: 895102. 